\title{
Complex Formation between the Postsynaptic Scaffolding Protein Gephyrin, Profilin, and Mena: A Possible Link to the Microfilament System
}

\author{
Torsten Giesemann, ${ }^{1}$ Günter Schwarz, ${ }^{2}$ Ralph Nawrotzki, ${ }^{3}$ Kerstin Berhörster, ${ }^{1}$ Martin Rothkegel, ${ }^{1}$ Kathrin Schlüter, ${ }^{1}$ \\ Nils Schrader, ${ }^{2,4}$ Hermann Schindelin, ${ }^{4}$ Ralf R. Mendel, ${ }^{2}$ Joachim Kirsch, ${ }^{3}$ and Brigitte M. Jockusch ${ }^{1}$ \\ ${ }^{1}$ Cell Biology, Zoological Institute, Technical University of Braunschweig, D-38092 Braunschweig, Germany, ${ }^{2}$ Institute for Plant Science, Technical \\ University of Braunschweig, D-38023 Braunschweig, Germany, ${ }^{3}$ Department of Anatomy and Cellular Neurobiology, University of Ulm, D-89069 Ulm, \\ Germany, and ${ }^{4}$ Department of Biochemistry and Center for Structural Biology, State University of New York at Stony Brook, Stony Brook, New York 11794- \\ 5115
}

Gephyrin is an essential component of the postsynaptic cortical protein network of inhibitory synapses. Gephyrin-based scaffolds participate in the assembly as well as the dynamics of receptor clusters by connecting the cytoplasmic domains of glycine and $\mathrm{GABA}_{\mathrm{A}}$ receptor polypeptides to two cytoskeletal systems, microtubules and microfilaments. Although there is evidence for a physical linkage between gephyrin and microtubules, the interaction between gephyrin and microfilaments is not well understood so far. Here, we show that neuronal gephyrin interacts directly with key regulators of microfilament dynamics, profilin I and neuronal profilin IIa, and with microfilament adaptors of the mammalian enabled (Mena)/vasodilator stimulated phosphoprotein (VASP) family, including neuronal Mena. Profilin and Mena/VASP coprecipitate with gephyrin from tissue and cells, and complex formation requires the E-domain of gephyrin, not the proline-rich central domain. Consequently, gephyrin is not a ligand for the proline-binding motif of profilins, as suspected previously. Instead, it competes with G-actin and phospholipids for the same binding site on profilin. Gephyrin, profilin, and Mena/VASP colocalize at synapses of rat spinal cord and cultivated neurons and in gephyrin clusters expressed in transfected cells. Thus, Mena/VASP and profilin can contribute to the postulated linkage between receptors, gephyrin scaffolds, and the microfilament system and may regulate the microfilament-dependent receptor packing density and dynamics at inhibitory synapses.

Key words: gephyrin; postsynaptic receptor dynamics; Mena; profilins; synaptic efficacy; VASP

\section{Introduction}

Neurotransmission at excitatory and inhibitory synapses requires the local concentration of receptor proteins at specialized plasma membrane domains. Clustering of the inhibitory glycine and $\mathrm{GABA}_{\mathrm{A}}$ receptors in the postsynaptic membrane depends on gephyrin expression (Pfeiffer et al., 1982; Schmitt et al., 1987; Kirsch and Betz, 1993). Gephyrin forms submembranous scaffolds and binds directly or indirectly to cytoplasmic loops of receptor subunits (Kneussel et al., 1999; Meier et al., 2001). The polypeptide of mammalian gephyrin comprises three modules: the N-terminal G-domain, a proline-rich central domain, and the C-terminal E-domain, respectively. Various isoforms are gener-

Received June 9, 2003; revised July 24, 2003; accepted July 24, 2003.

This work was supported by the Deutsche Forschungsgemeinschaft, the "Fritz-Thyssen-Stiftung," and the "Fonds der Chemischen Industrie". We thank Jens Paulukat (Braunschweig) for providing various gephyrin expression vectors and Drs. Walter Witke (Monterotondo, Italy) and Jürgen Wehland (Braunschweig) for the profilin Ila cDNA and for monoclonal antibodies to VASP and Mena, respectively. Technical help by Tanja Otte (Braunschweig), Maria Manz (Ulm), and Ingeborg Vogel (Ulm) is gratefully acknowledged.

Correspondence should be addressed to Brigitte M. Jockusch, Cell Biology, Zoological Institute, Technical University, D-38092 Braunschweig, Germany. E-mail: bmj@tu-bs.de.

R. Nawrotzki's and T. Kirsch's present address: Institute for Anatomy and Cell Biology, University of Heidelberg, 69120 Heidelberg, Germany.

K. Schlüter's present address: Clontech, BD Biosciences, 69126 Heidelberg, Germany.

Copyright $\odot 2003$ Society for Neuroscience $\quad$ 0270-6474/03/238330-10\$15.00/0 ated by RNA splicing (Prior et al., 1992; Ramming et al., 2000), possibly relating to the fact that gephyrin is a dual-function protein, contributing to molybdenum cofactor biosynthesis in addition to receptor clustering (Feng et al., 1998; Stallmeyer et al., 1999; Ramming et al., 2000).

At the postsynaptic membrane of inhibitory synapses, gephyrin scaffolds are thought to interact with the submembranous cytoskeleton. The packing density of glycine receptors in postsynaptic clusters depends on the integrity of the cytoskeletal elements (Kirsch and Betz, 1995; Kneussel and Betz, 2000). Although depolymerization of neuronal microtubules reduced receptor packing density, microfilament breakdown increased it (Kirsch and Betz, 1995). Because the packing density in turn modulates receptor-ligand affinities, the cytoskeleton may play a pivotal role in regulating the efficacy of inhibitory synaptic transmission, and microtubules and microfilaments could act antagonistically in this regulatory process.

Several molecular links between gephyrin and the microtubular cytoskeleton have already been identified. Gephyrin was found to bind to microtubules and tubulin (Kirsch et al., 1991; Ramming et al., 2000). Furthermore, complex formation of gephyrin with the microtubule motor dynein has been demonstrated (Fuhrmann et al., 2002). In contrast, a direct interaction 
of gephyrin with microfilament components was less well documented. In one report, an in vitro interaction between gephyrin and the microfilament protein profilin was demonstrated (Mammoto et al., 1998). Profilins are small proteins involved in the regulation of actin dynamics (Carlsson et al., 1976). In mammals, several profilin isoforms were identified. Although profilin I is expressed ubiquitously, the isoform IIa is found primarily in neurons (Honoré et al., 1993; Giesemann et al., 1999; Di Nardo et al., 2000). Profilins bind to G-actin, to the signaling molecule phosphatidylinositol-4,5-bisphosphate $\left(\mathrm{PIP}_{2}\right)$, and to various polyproline-rich proteins (Schlüter et al., 1997), including proteins of the mammalian enabled (Mena)/vasodilator stimulated phosphoprotein (VASP) family (Reinhard et al., 1995). These proteins are microfilament adaptors involved in the recruitment of profilin/actin complexes to membranes engaged in actin filament dynamics (Hüttelmaier et al., 1998; Bear et al., 2002) and in the suprastructural organization of actin filaments (Reinhard et al., 1992; Gertler et al., 1996; Hüttelmaier et al., 1999). Hence, profilins and Mena/VASP proteins are both considered essential for submembranous actin filament generation and organization.

Here, we show that gephyrin, profilins, and Mena/VASP proteins colocalize at synapses and in gephyrin clusters generated in transfected cells. Complexes containing these components can be precipitated from brain extracts and cell lysates, and in vitro, recombinant gephyrin interacts with Mena/VASP and profilins. We determined the gephyrin module mediating these interactions and show that gephyrin and G-actin compete for the same binding site on profilin. Thus, we identified components of the postulated molecular link between gephyrin and microfilaments that in cooperation with microtubules may regulate the receptor packing densities at inhibitory postsynaptic membrane specializations.

\section{Materials and Methods}

Plasmids. Full-length gephyrin cDNA (aa 1-736; gene bank accession no. X66366) was generated by PCR and subcloned into the vectors pQE-30 (Qiagen, Hilden, Germany), pEGFP-C2 (Clontech, Heidelberg, Germany), pcDNA3 (Invitrogen, Groningen, Netherlands), and pcFlag (SigmaAldrich, Deisenhofen, Germany) and into a modified pcDNA3 (pcBiPro), comprising a DNA sequence coding for an epitope tag recognized by a monoclonal antibody raised against birch profilin (a-BiPro 4A6) (Rüdiger et al., 1997) (Fig. 1). Full-length gephyrin, gephyrin G-domain (aa 1-175), G-domain with central C-domain (aa 1-310), and E-domain (aa 318-736) were generated by PCR and cloned into pcDNA3, and, additionally, full-length gephyrin and the E-domain into pcFlag (Fig. 1). cDNAs coding for mouse profilin I (mPFN I; accession no. X14425) and human profilin I (hPFN I; accession no. J03191) were cloned as NcoIEcoRI DNA fragments into pET $28 \mathrm{a}(+)$ (Novagen, Bad Soden, Germany) (Fig. 1). The prokaryotic expression plasmid pMW172-mPFN IIa was used for expression of mouse profilin IIa (mPFN IIa; accession no. AJ272203) (W. Witke, European Molecular Biology Laboratory, Monterotondo, Italy). Additionally, mPFN I and IIa cDNAs were inserted into pcFlag. Point mutations within the hPFN I cDNA (Y59A and H133S) were introduced by site-directed mutagenesis (Stratagene, Amsterdam, The Netherlands) and cloned in pET $28 \mathrm{a}(+)$. Recombinant VASP was expressed using the prokaryotic expression vector pQE30-BiPro-VASP (Hüttelmaier et al., 1999). The gephyrin binding motif (residues 378426) (Meyer et al., 1995) of the glycine receptor $\beta$-subunit (accession no. AJ310839; nucleotides 1132-1278) was PCR cloned into pTYB2 (New England Biolabs, Schwalbach, Germany). All constructs were verified by DNA sequencing (ABI Prism 310 Sequencer, PE Biosystems, Weiterstadt, Germany).

Antibodies. For immunofluorescence, immunoprecipitation, or immunoblotting, the following antibodies were applied: monoclonal antibodies against rat synaptophysin (Sigma-Aldrich), human VASP (J. Wehland, German Research Center for Biotechnology (GBF), Braun-
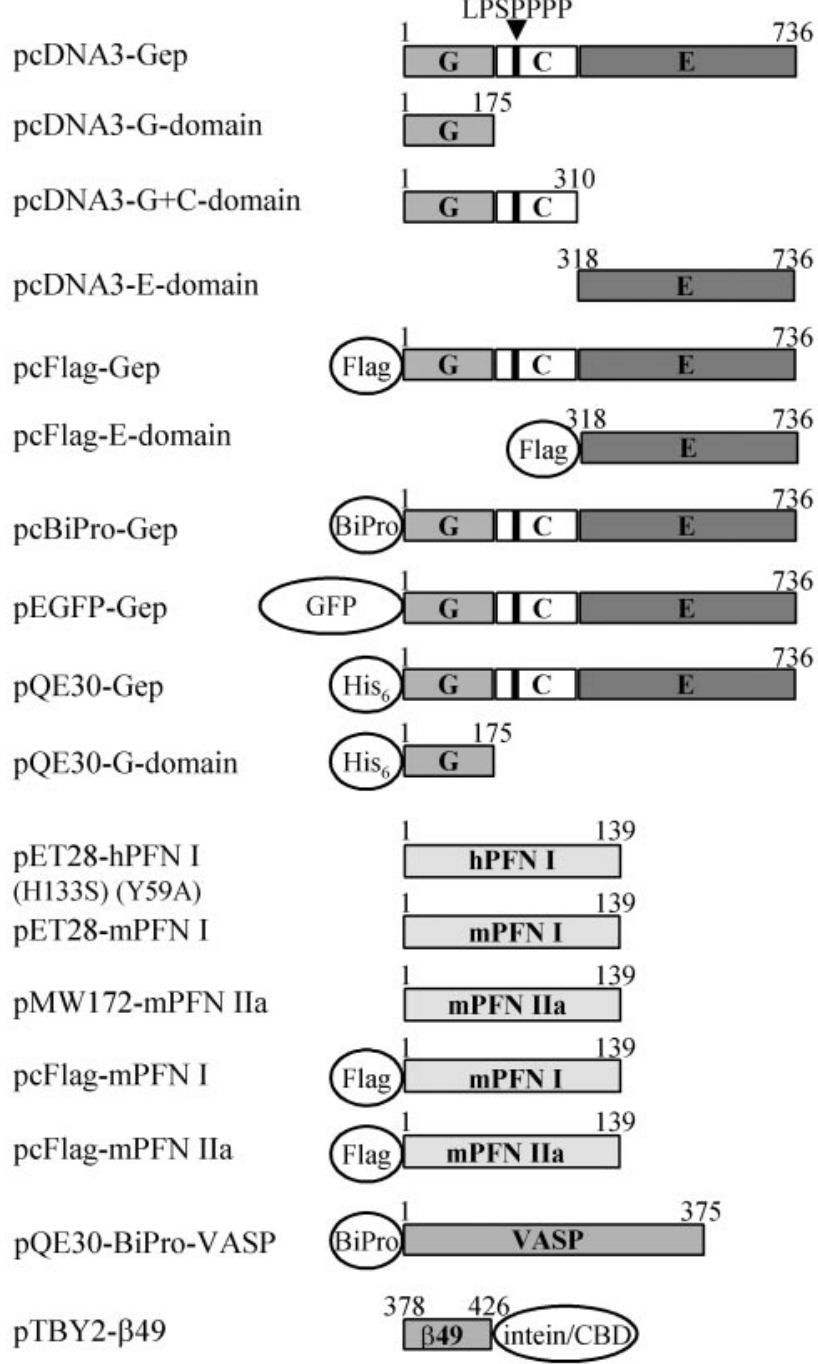

Figure 1. Prokaryotic and eukaryotic expression constructs and schematic representation of the encoded recombinant proteins: rat gephyrin (Gep) and its fragments, human profilin I (hPFN I), including mutants $\mathrm{H} 133 \mathrm{~S}$ and Y59A, respectively, murine profilins I (mPFN I) and Ila (mPFN Ila), VASP, and the $\beta$-subunit of the glycine receptor ( $\beta 49)$. The diagram of the gephyrin polypeptide (Gep) organization shows the G-domain $(G)$, the central domain $(C)$ with the proline-rich sequence, and the E-domain (E) (Prior et al., 1992; Stallmeyer et al., 1999). Numbers refer to the amino acid residues. LPSPPPP, The proline-rich sequence within the central domain. The positions of the fused epitope tags (Flag, BiPro), the His - -tag, and the polypeptides GFP and intein with chitin binding domain (CBD) are indicated.

schweig) and murine Mena (J. Wehland, GBF Braunschweig, and BD Transduction Laboratories, Heidelberg, Germany), a monoclonal antibody against rat gephyrin (BD Transduction Laboratories) and rabbit serum against rat gephyrin (our own), monoclonal antibodies against monomeric actin (Gonsior et al., 1999), Flag tag (Sigma-Aldrich) and BiPro tag (Rüdiger et al., 1997), and different antibodies against profilin: a monoclonal against bovine profilin I that is specific for profilin I and recognizes many mammalian profilins but not rodent profilins (Mayboroda et al., 1997), and rabbit antisera against human or bovine profilin recognizing both profilin I and IIa, which also react with the corresponding rodent profilins (Cytoskeleton, Denver, CO; and our own). Cy-3conjugated rabbit-anti-(mouse IgG) or sheep anti-(rabbit IgG) (SigmaAldrich), tetramethylrhodamine isothiocyanate (TRITC)-conjugated rabbit anti-(mouse IgG) (Sigma-Aldrich), Alexa Fluor 488-conjugated rabbit-anti-(mouse IgG), and peroxidase-conjugated rabbit anti-(mouse IgG) (Sigma-Aldrich) served as secondary antibodies.

Preparation of tissue and cells for fluorescence analysis. Freshly prepared 
rat spinal cord tissue was frozen in liquid nitrogen and sectioned at $8 \mu \mathrm{m}$ in the transverse plane. Unfixed sections, mounted on glass coverslips, were blocked in $5 \%(\mathrm{v} / \mathrm{v})$ normal goat serum and processed for immunofluorescence as described (Kirsch and Betz, 1993).

Spinal cord was prepared from rat at embryonic day 14 (E14). After removal of the dura, cells were dissociated by trituration and seeded in Neurobasal medium (Invitrogen, Karlsruhe, Germany) supplemented with $2 \%$ (v/v) B27, $2 \mathrm{~mm}$ glutamine, $25 \mathrm{~mm}$ pyruvate, $50 \mathrm{IU} / \mathrm{ml}$ penicillin, and 50 $\mu \mathrm{g} / \mathrm{ml}$ streptomycin (Invitrogen) onto poly-L-ornithine-covered glass coverslips as described (Kirsch and Betz, 1993). After $15 \mathrm{~d}$ in culture, cells were fixed with $95 \%$ methanol $/ 5 \%$ acetic acid $(\mathrm{v} / \mathrm{v})\left(5 \mathrm{~min}\right.$ at $\left.-80^{\circ} \mathrm{C}\right)$ and processed for immunofluorescence. The cell lines human cervix carcinoma (HeLa), human embryonic kidney 293 (HEK293), and rat phaechromocytoma (PC12) were cultured according to standard protocols. HeLa cells, grown on glass coverslips, were cotransfected with pEGFP-gep and pc-FlagmPFN I or pc-Flag-mPFN IIa by calcium phosphate precipitation. Thirtysix hours after transfection, cells were fixed after washing.

All cells were fixed either with $2 \%$ formaldehyde or with $0.5 \mathrm{~mm}$ dithiobis (succinimidyl propionate) (DSP; Pierce, Rockford, IL), (Hüttelmaier et al., 1999). After detergent extraction, primary and secondary antibodies were applied according to standard protocols. Samples were examined using a Leica OMRE laser scanning confocal microscope or a Zeiss Axiophot microscope equipped with epifluorescence, and pictures were processed using the program Metamorph (Universal Imaging, West Chester, PA).

Immunoprecipitation and pull-down experiments. Brains from adult rats (female CD; Charles River Laboratories, Sulzfeld, Gemany) or mice [ICR (CD-1); Harlan-Winkelmann, Borchen, Germany] were excised, frozen in liquid nitrogen, and homogenized. Two hundred milligrams of powder were resuspended in $1 \mathrm{ml}$ extraction buffer $(20 \mathrm{~mm}$ Tris- $\mathrm{HCl}, \mathrm{pH}$ 8.0, $100 \mathrm{~mm} \mathrm{NaCl}, 0.5 \mathrm{~mm}$ EDTA, protease inhibitors) and triturated by sonication at $4^{\circ} \mathrm{C}$. The extract was cleared by centrifugation (two times for $45 \mathrm{~min}$ each; $30,000 \times g ; 4^{\circ} \mathrm{C}$ ). For affinity pull-down experiments, the gephyrin binding sequence of the glycine receptor $\beta$ subunit $(\beta 49)$ was expressed as a recombinant fusion protein with intein and a chitinbinding domain and purified on chitin beads (IMPACT protein expression system, New England Biolabs). Thirty-five microliters of chitin beads saturated with this fusion protein were incubated with $1 \mathrm{ml}$ brain extract (protein concentration $10 \mathrm{mg} / \mathrm{ml}$ ) overnight. Alternatively, these beads were incubated with purified profilin I, preincubated with excess poly-L-proline [molecular weight (MW) $\sim 5400 \mathrm{Da}$; Sigma-Aldrich] or gephyrin (500 pmol each), or both. Uncoupled beads or beads containing only the intein/chitin binding domain tag served as a negative control. Beads were sedimented after washing and processed for SDS-PAGE. For coimmunoprecipitation of gephyrin with ligands, $6 \mu \mathrm{l}$ of gephyrin antiserum was incubated with the brain extracts as described above. Precipitation was performed with Protein-G-Sepharose (Amersham Biosciences, Freiburg, Germany).

For analysis of protein complexes from cell lysates, HeLa and HEK293 cells were transiently transfected with the appropriate vectors by calcium phosphate precipitation and lysed, and immunoprecipitates were obtained from clarified supernatants as above. In some experiments, we treated the cells with the membrane permeant cross-linker DSP before lysis as described (Hüttelmaier et al., 1998; Giesemann et al., 1999), but this was not obligatory for complex preservation.

In vitro transcription/translation, protein expression and purification. $\left[{ }^{35} \mathrm{~S}\right]$-met-labeled gephyrin and deletion fragments were synthesized by in vitro transcripition/translation using the TNT-coupled reticulocyte lysate system (Promega, Heidelberg, Germany). Recombinant rat gephyrin, gephyrin G-domain, and VASP were obtained from Escherichia coli (M15, Qiagen), transformed with the vector pQE30-Gep, pQE30-Gdomain (Stallmeyer et al., 1999), or pQE30-BiPro-VASP (Hüttelmaier et al., 1999; Harbeck et al., 2000), respectively (compare Fig. 1). After induction of expression, proteins were purified as described (Qiagen) using an imidazole gradient for protein elution. Gephyrin E-domain was obtained from holo-gephyrin by limited proteolysis with trypsin and subsequent purification using anion exchange (MonoQ, Amersham Biosciences) and size exclusion chromatography (Superdex200, Amersham Biosciences). Rabbit skeletal muscle actin was prepared as described pre- viously (Giehl et al., 1994). Recombinant human and mouse profilins were produced in E. coli BL21 (DE3) (Stratagene), which was transformed with the expression vectors described above (Fig. 1). Expression and purification of these proteins were performed by poly-L-proline affinity chromatography, using a two-step urea gradient for elution (Giehl et al., 1994; Wittenmayer et al., 2000). For the mutant hPFN I-H133S, much weaker binding to the polyproline affinity column was observed. The purity of all protein preparations was better than $95 \%$, as judged by Coomassie Blue-stained SDS-gel profiles. Protein concentration was quantified by the Bradford assay, using BSA as a standard.

Solid-phase binding and competition assays. Dot overlays were performed as described (Giesemann et al., 1999). Recombinant gephyrin, mouse profilin I and IIa, and BSA were adsorbed to nitrocellulose membrane and unspecific binding sites were blocked. In vitro translated proteins were diluted in TBST [ $20 \mathrm{~mm}$ Tris, pH 7.6, $140 \mathrm{~mm} \mathrm{NaCl}, 0.1 \%$ (v/v) Tween 20] containing $1 \%(\mathrm{w} / \mathrm{v}) \mathrm{BSA}$ and $20 \mathrm{~mm} \beta$-mercaptoethanol and incubated with the membrane for $4 \mathrm{hr}$ at room temperature. After washing, bound proteins were detected by autoradiography. For demonstration of the ligand-binding capacity of membrane-bound profilins, the overlay reaction was performed with purified mouse profilin I and actin in G-buffer (5 mM Tris- $\mathrm{HCl}$, pH 8.3, $1 \mathrm{~mm}$ ATP, $0.2 \mathrm{~mm} \mathrm{CaCl}_{2}, 2 \mathrm{~mm}$ dithioerythritol), and bound actin was detected with a G-actin-specific antibody (Gonsior et al., 1999) and HRP-coupled secondary antibody. For ELISAs, microtiter plates (Microlon, Greiner, Frickenhausen, Germany) were coated with 25 pmol per well BSA (control), recombinant gephyrin, the proteolytically obtained E-domain, or the recombinant G-domain, respectively, in $50 \mathrm{~mm} \mathrm{NaHCO}$, pH 9.6, washed repeatedly with buffer A [0.05\% (v/v) Tween 20 in PBS, pH 7.4] and blocked with $1 \%(\mathrm{w} / \mathrm{v}) \mathrm{BSA}$, before application of the recombinant protein human profilin I or its mutants Y59A and H133S, respectively, in varying amounts $(0-100 \mathrm{pmol})$. For competition assays, per reaction, $25 \mathrm{pmol}$ of profilin I was preincubated with varying amounts $(0-100 \mathrm{pmol})$ of actin, poly-L-proline, and $\mathrm{PIP}_{2}$ (Boehringer, Ingelheim, Germany), respectively, for $30 \mathrm{~min}$ at room temperature. $\mathrm{PIP}_{2}$ micelles were prepared as described (Hüttelmaier et al., 1998). Binding of VASP to microwelladsorbed gephyrin was tested with recombinant BiPro-VASP. Bound profilin or VASP was monitored with the anti-profilins or the BiProantibody, respectively, as specified in the Figure legends. For quantitation, 2,2'-azinobis(3-ethylbenzthiazoline-sulfonic acid) (SigmaAldrich) was used as a substrate, and color development was monitored in an ELISA reader (Dynatech, Denkendorf, Germany).

\section{Results}

\section{Profilin and gephyrin colocalize in inhibitory synapses of spinal cord and cultured neurons}

To characterize the potential interaction between profilins and gephyrin, we analyzed their localization in tissue sections and cultured neuronal cells. First, we detected that the synaptic marker protein synaptophysin (Fig. $2 A$ ) colocalizes with profilin (Fig. $2 A^{\prime}$ ) in bona fide synaptic structures on rat spinal cord sections. In this case, the merged images reveal a very high degree of superposition illustrating a general enrichment of both proteins in synapses (Fig. $\left.2 A^{\prime \prime}\right)$. The distribution of gephyrin, a marker for inhibitory synapses, was analyzed in sections of rat spinal cord enriched in inhibitory synapses. As shown in Figure 2, $B$ and $B^{\prime}$, gephyrin also colocalizes with profilin; however, the profilin distribution on these sections appeared different. Although only few profilin-positive spots were found that were negative for synaptophysin, there were numerous profilinpositive densities lacking gephyrin staining, indicating the additional presence of noninhibitory synapses on these sections (Fig. 2, compare $A, A^{\prime \prime}$ with $\left.B, B^{\prime \prime}\right)$. Neurons cultured from rat spinal cord yielded analogous results. All synaptophysin- and gephyrinpositive densities displayed prominent signals for profilin (Fig. $\left.2 C, C^{\prime \prime}, D, D^{\prime \prime}\right)$, but there were more profilin-positive than gephyrin-positive densities (Fig. $2 D, D^{\prime \prime}$ ). These results show that profilin is generally enriched in synapses and illustrate that inhib- 

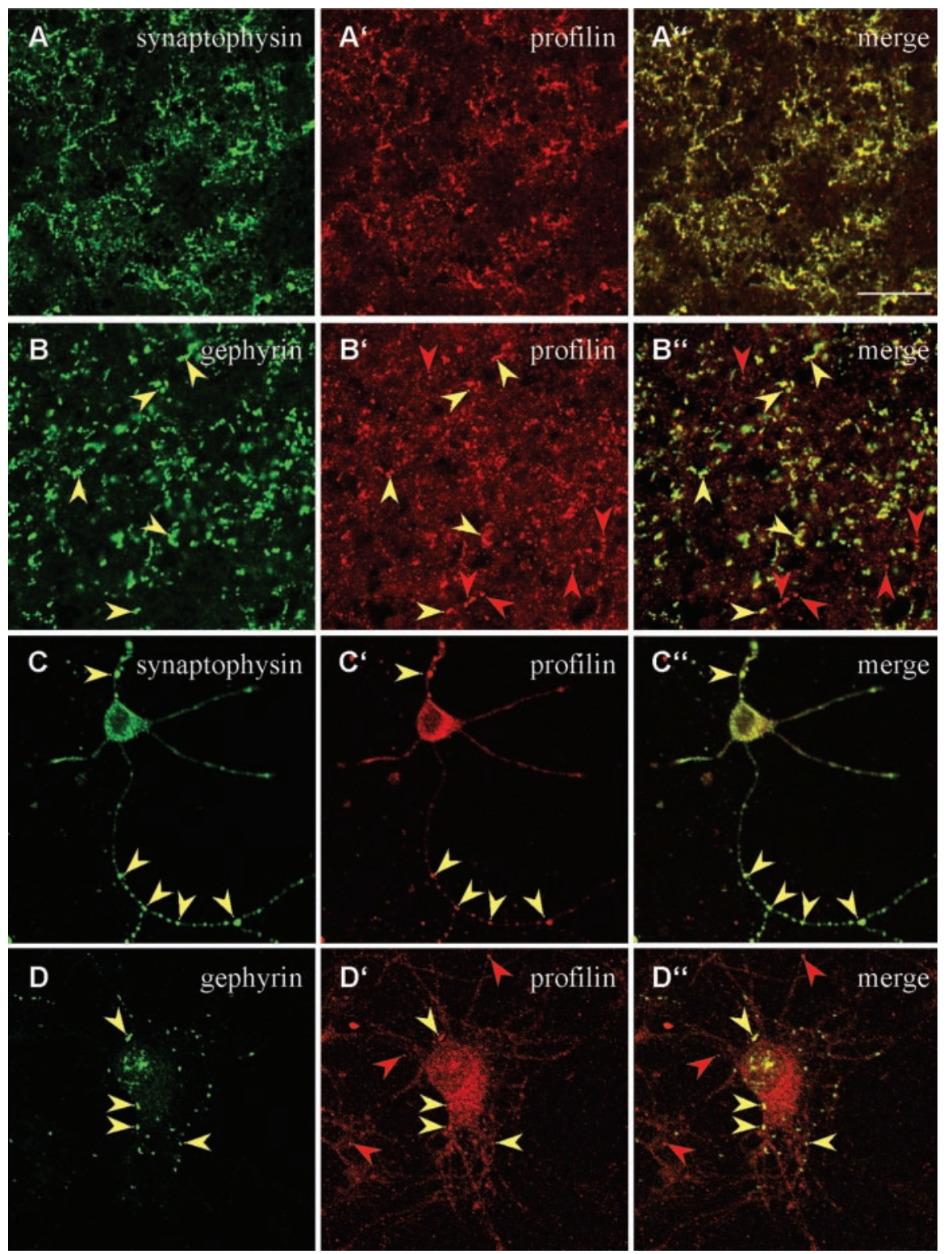

Figure 2. Profilin and gephyrin colocalize at rat spinal cord synaptic structures. Profilin was stained with a polyclonal antibody recognizing rodent profilins I and II. $A, A^{\prime \prime}$, Double immunofluorescence revealing synaptophysin, a synapse marker ( $A$, green) with profilin $\left(A^{\prime}\right.$, red). The merged image $\left(A^{\prime \prime}\right)$ shows that all synapses are enriched for profilin. $B, B^{\prime \prime}$, Corresponding double labeling for gephyrin, the postsynaptic marker of inhibitory neurons, with a monoclonal anti-gephyrin $\left(B\right.$, green) and profilin $\left(B^{\prime}\right.$, red). This section was taken from a region of the spinal cord especially rich in inhibitory synapses. Note that all gephyrin-positive densities are also enriched in profilin $\left(B, B^{\prime \prime}\right.$, yellow arrowheads), but many profilin-positive speckles $\left(B^{\prime}, B^{\prime \prime}\right.$, red arrowheads) lack gephyrin staining. $C, C^{\prime \prime}, A$ cultured spinal cord neuron displaying numerous synaptophysin-positive dendritic synapses ( $C$, green) that are also enriched for profilin ( $C^{\prime}$, red and merged image, $C^{\prime \prime}$, yellow, arrowheads). $D, D^{\prime \prime}$, Double immunofluorescence of gephyrin $(D$, green) and profilin $\left(D^{\prime}\right.$, red) of a cultured rat spinal cord neuron. Gephyrin and profilin show colocalization $\left(D, D^{\prime \prime}\right.$, yellow arrowheads), but there are numerous profilin-positive densities not positive for gephyrin $\left(D^{\prime}, D^{\prime \prime}\right.$, red arrowheads). Scale bars, $10 \mu \mathrm{m}$. brain extracts. These results were corroborated by data obtained with purified components: mouse profilin I was efficiently precipitated together with gephyrin by these beads. Some batches of the beads coated with the gephyrin-binding sequence of the glycine receptor had a tendency to bind recombinant profilin I in the absence of gephyrin; however, this could be greatly reduced by preincubating profilin I with an excess of poly-L-proline before the addition of beads (Fig. $3 B$ ). Taken together, these results indicated that gephyrin mediated the interaction of profilin with the glycine receptor $\beta$ subunit and that the polyproline binding site on profilin was not involved in the complex formation between profilin and gephyrin.

This complex formation was investigated further by immunoprecipitation performed with tissue and cell lysates and in ELISAs with recombinant components. Figure $4 A$ shows that anti-gephyrin precipitates gephyrin together with profilin from rat and mouse brain extracts. For coprecipitation studies from cells, we used HeLa cells transiently transfected with a tagged gephyrin (BiPro-gephyrin) and HeLa cells double transfected with BiProgephyrin and Flag-tagged mouse profilin I or Flag-tagged mouse profilin IIa. Figure $4 B$ shows that in precipitates obtained with the BiPro-antibody, the tagged gephyrin was precipitated together with the endogenous HeLa cell (human) profilin but also with the tagged mouse profilins I and IIa. These data imply a physical interaction between gephyrin and profilins in neuronal and non-neuronal cells.

The binding of gephyrin to both profilin isoforms was further analyzed in ELISAs. Figure $4 C$ shows that the purified, recombinant proteins also interact in such assays: profilins I and IIa bind to microwell-adsorbed gephyrin.

\section{The E-domain of gephyrin mediates profilin binding}

Next, we identified the gephyrin domain involved in this interaction. To this end, we performed solid-phase binding assays itory synapses contain a high concentration of both gephyrin and profilin.

\section{Gephyrin and profilins form complexes in brain tissue, transfected cells, and in vitro}

To investigate whether profilins were able to form complexes with gephyrin, we performed coprecipitation experiments and studied the possible direct interaction between the components (Fig. 3). In pull-down experiments using beads coated with the gephyrin-binding sequence of the glycine receptor $\beta$ subunit, rat (Fig. $3 A$ ) and mouse (Fig. $3 A^{\prime}$ ) profilins were coprecipitated from with in vitro translated $\left[{ }^{35} \mathrm{~S}\right]$-methionine-labeled gephyrin, the G-domain, with or without the central domain, and the E-domain, respectively (Fig. 1 shows the domain organization of gephyrin). Synthesis of all products with the expected size was verified in autoradiographs obtained after SDS-PAGE (Fig. 5A), and aliquots were added to spotted recombinant gephyrin, mouse profilin I, or mouse profilin IIa. The ligand-binding ability of immobilized profilin was verified by overlay reaction with purified muscle G-actin and anti-actin (Fig. 5A', left column). The weak spot seen for gephyrin overlaid with actin was considered not significant (Fig. $5 A^{\prime}$ ). Binding of $\left[{ }^{35} \mathrm{~S}\right]$-methionine-labeled 
A Cosedimentation from rat and mouse brain

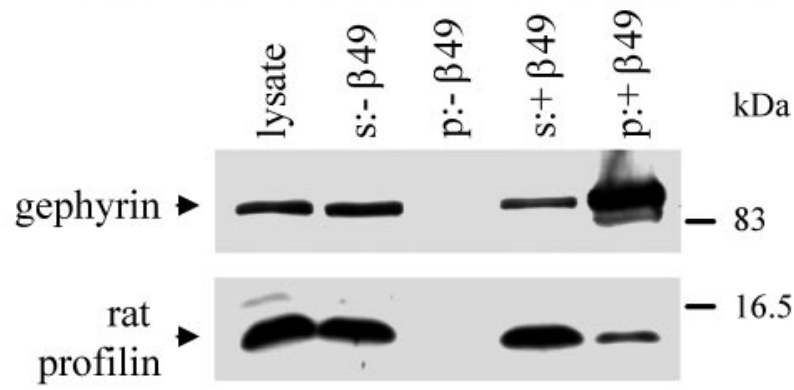

$\mathbf{A}^{6}$

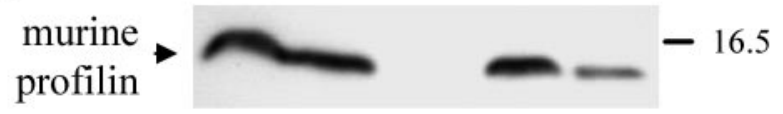

B Cosedimentation of recombinant proteins

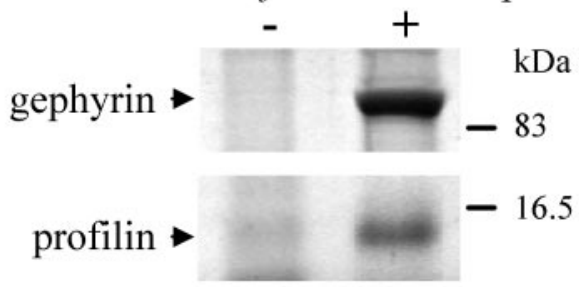

Figure 3. Gephyrin and profilins form complexes in cells and interact in vitro. $A, A^{\prime}$, Affinity precipitation of profilin with gephyrin from rat $(A)$ and mouse $\left(A^{\prime}\right)$ brain extracts, using beads coated with the gephyrin-binding sequence of the glycine receptor $\beta$-subunit ( $\beta 49)$. Aliquots of extracts were incubated with beads, sedimented, and subjected to SDS-PAGE and immunoblotting, using polyclonal antibodies against gephyrin and both profilin isoforms. Lysate, Gephyrin and profilin shown in the total extracts; s:- $\beta 49$ and p:- $\beta 49$, supernatant and pellet of control beads coated only with the intein/chitin binding domain fusion peptide (see Material and Methods); s: $+\beta 49$ and $p:+\beta 49$, analogous probes obtained with beads coated with the gephyrin-binding sequence of the glycine receptor $(\beta 49)$. $B$, Cosedimentation of the $\beta 49$ beads and recombinant profilin in the presence $(+)$ or absence $(-)$ of gephyrin. The pull-down assay was performed with recombinant gephyrin and recombinant profilin I, preincubated with an excess of poly---proline. SDS-PAGE and immunoblotting were as indicated in $A$ and $A^{\prime}$.

gephyrin and its fragments to membrane-adsorbed gephyrin and both profilins was detected by autoradiography (Fig. $5 A^{\prime}$, right panels). Both gephyrin and the E-domain reacted strongly with the profilin isoforms and with gephyrin. Binding of the G-domain, with or without the central domain, to immobilized profilin was very weak and considered not significant, whereas both of these fragments bound to membrane-immobilized gephyrin, reflecting the well known self association (Fig. 5 $A^{\prime}$ ). Immunoprecipitation studies with transfected cells corroborated these results. From lysates of HeLa cells transfected with Flagtagged gephyrin or Flag-tagged E-domain, endogenous HeLa profilin was coprecipitated with both proteins by the Flag antibody (Fig. 5B). Corresponding data were also obtained in an ELISA: recombinant human profilin I, added to either gephyrin or the E-domain adsorbed to microwells, was retained by both proteins (Fig. 5C). Analogous experiments with the G-domain showed no binding of profilin to this module (data not shown).

Gephyrin, $\mathrm{PIP}_{2}$ and G-actin compete for the same binding site on profilins

To identify the ligand binding site on profilins involved in this interaction, we used competition ELISAs. Recombinant human profilin I was preincubated with either one of the acknowledged partners for the three major binding sites of profilin, namely

\section{A Co-IP from rat and mouse brain}

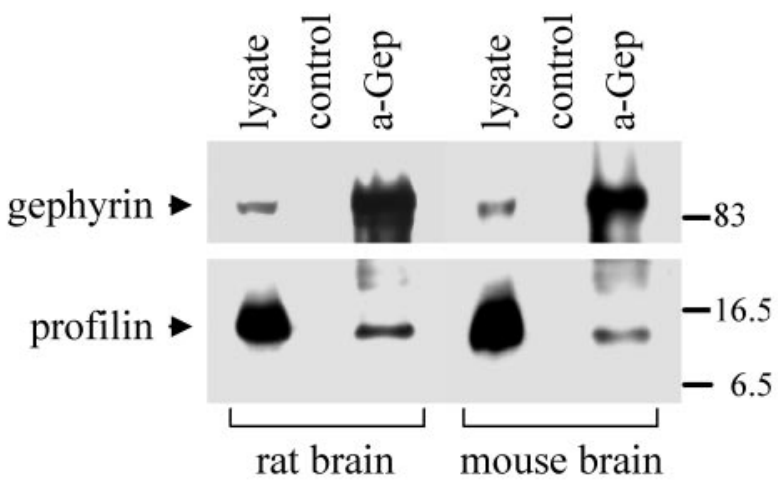

B Co-IP from HeLa-cells
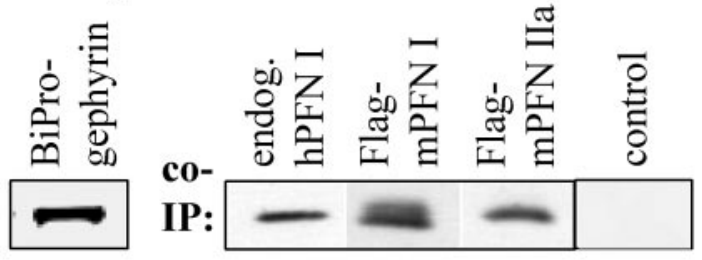

C

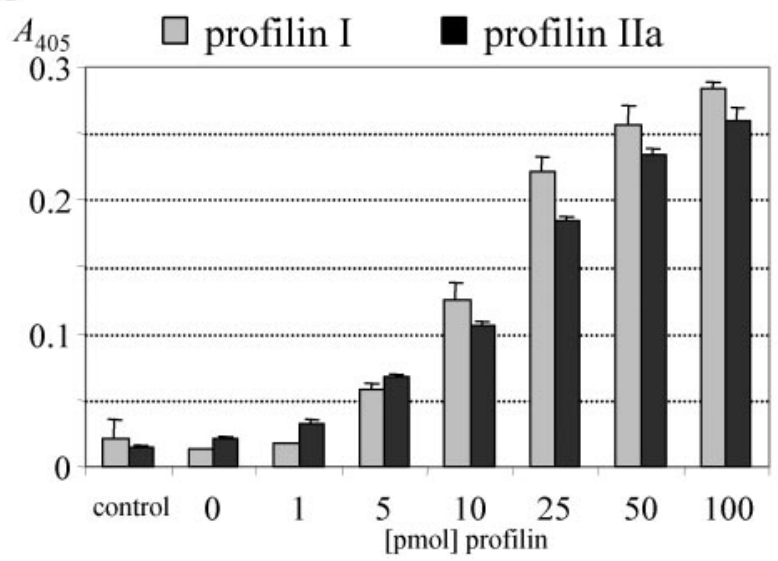

Figure 4. Profilin and gephyrin interact in brain tissue, cells, and in vitro. A, Immunoprecipitation from rat and mouse brain extracts, performed with the polyclonal anti-gephyrin (a-Gep). Sediments were subjected to SDS-PAGE and immunoblotting with a polyclonal anti-profilin. Control, Aliquot without anti-gephyrin. $B$, Immunoprecipitation of gephyrin and profilin from cell lysates. HeLa cells were transiently single transfected with BiPro-tagged gephyrin or double transfected with either Flag-tagged mouse profilin I or lla, respectively. Immunoprecipitation was performed with the BiPro antibody, and immunoblots were obtained after SDS-PAGE. Transfected profilins were detected by Flag antibodies as indicated; endogenous profilin I was detected with a monoclonal profilin antibody. Note that endogenous human profilin I and both isoforms of transfected mouse profilins coimmunoprecipitated with transfected gephyrin. Control, Immunoblot derived from nontransfected HeLa cells. Lysates were treated with the BiPro antibody and protein G-Sepharose; sediments were subjected to SDS-PAGE and probed for endogenous profilin I. C, ELISA to show direct interaction between gephyrin and either profilin I or lla. Twenty-five picomoles of recombinant gephyrin were adsorbed to ELISA wells and incubated with increasing amounts of recombinant profilin I (gray bars) and Ila (black bars) and a polyclonal anti-profilin recognizing both profilin isoforms. Note that both profilins bind to gephyrin with comparable affinity. Control, BSA (25 pmol) was immobilized and incubated with 100 pmol of mPFN I or mPFN Ila.

poly-L-proline, G-actin, or $\mathrm{PIP}_{2}$, and subsequently added to microwells coated with recombinant gephyrin. Binding of human profilin I was monitored with the monoclonal anti-profilin. The molar ratio of gephyrin and potential competitors varied between 


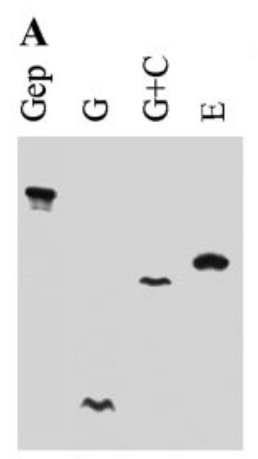

$\mathbf{A}^{6}$

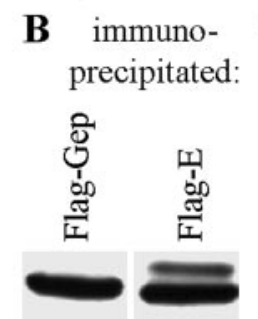

coprecipitated profilin:

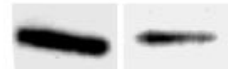

C
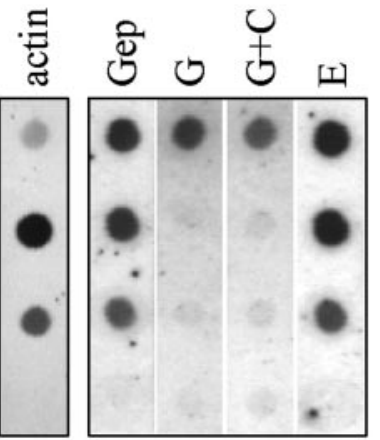

BSA
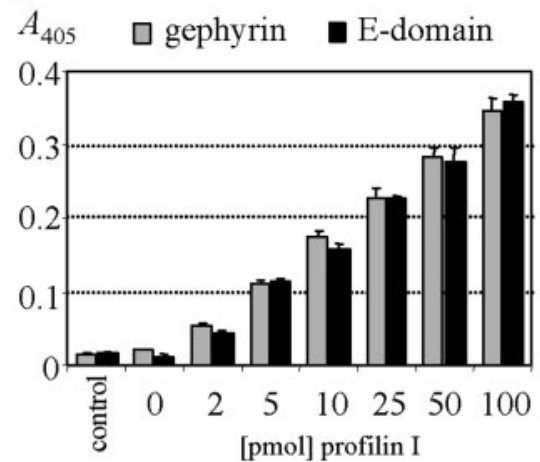

Figure 5. The E-domain of gephyrin interacts with profilins. $A, A^{\prime}$, Overlay assays with in vitro translated, $\left[{ }^{35} \mathrm{~S}\right]$-Met-labeled proteins. Gep, Gephyrin; E, G, E+C, G+C, corresponding deletion fragments of gephyrin (cf. Fig. 1). $A$, Translation of polypeptides with the expected size was controlled by SDS-PAGE and autoradiography. $A^{\prime}$, Binding of purified G-actin (left panel) and in vitro synthesized proteins (right panel, top) to membrane-adsorbed recombinant partners (left). BSA, Bovine serum albumin. Bound proteins were detected with specific antibodies (G-actin) or by autoradiography (others), respectively. Note that gephyrin interacts with itself and with both profilin isoforms and that binding is confined to the E-domain. $B$, Immunoblots obtained from HeLa cells transfected with Flag-tagged gephyrin or its E-domain. Immunoprecipitates were obtained with Flag antibody and probed with monoclonal anti-Flag and antiprofilin, demonstrating that the E-domain is sufficient to precipitate HeLa cell profilin I. C, ELISA with microwell-adsorbed recombinant gephyrin (gray bars) or the proteolytically derived E-domain (black bars; 25 pmol each) and soluble recombinant human profilin I. Binding of profilin was monitored with the monoclonal anti-profilin. Control was BSA (25 pmol) adsorbed to ELISA wells and incubated with $100 \mathrm{pmol}$ of profilin. Note that in this assay the proteolytic fragment comprising the E-domain is as efficient in binding profilin as intact gephyrin.

0 and a fourfold molar excess of the competitors. As shown in Figure $6 \mathrm{~A}$, addition of poly-L-proline, even in large excess, had no significant effect on the binding of profilin to gephyrin, although in corresponding ELISAs we could demonstrate the successful masking of the polyproline binding site on profilin by poly-Lproline incubation (data not shown). These results corroborated the data obtained with beads coated with the gephyrin-binding sequence of the glycine receptor (compare Fig. $3 B$ ). In contrast, preincubation with either $\mathrm{G}$-actin or $\mathrm{PIP}_{2}$ resulted in a dosedependent decrease of profilin binding to gephyrin (Fig. 6A). At fourfold molar excess, both of these ligands reduced the profilingephyrin interaction to $<20 \%$ of the control value. These data indicated that gephyrin binds to the actin $/ \mathrm{PIP}_{2}$-binding site on profilins but not to the polyproline-binding site.

To corroborate these results, human profilin I mutants were used in analogous ELISAs. Previously, we had shown that the hydrophobic amino acid residue in position 59 is crucial for the interaction of bovine (F59) and human (Y59) profilin I with actin. Replacing these residues by alanine reduces the affinity for $\mathrm{G}$-actin by more than a factor of 10 , whereas the binding of
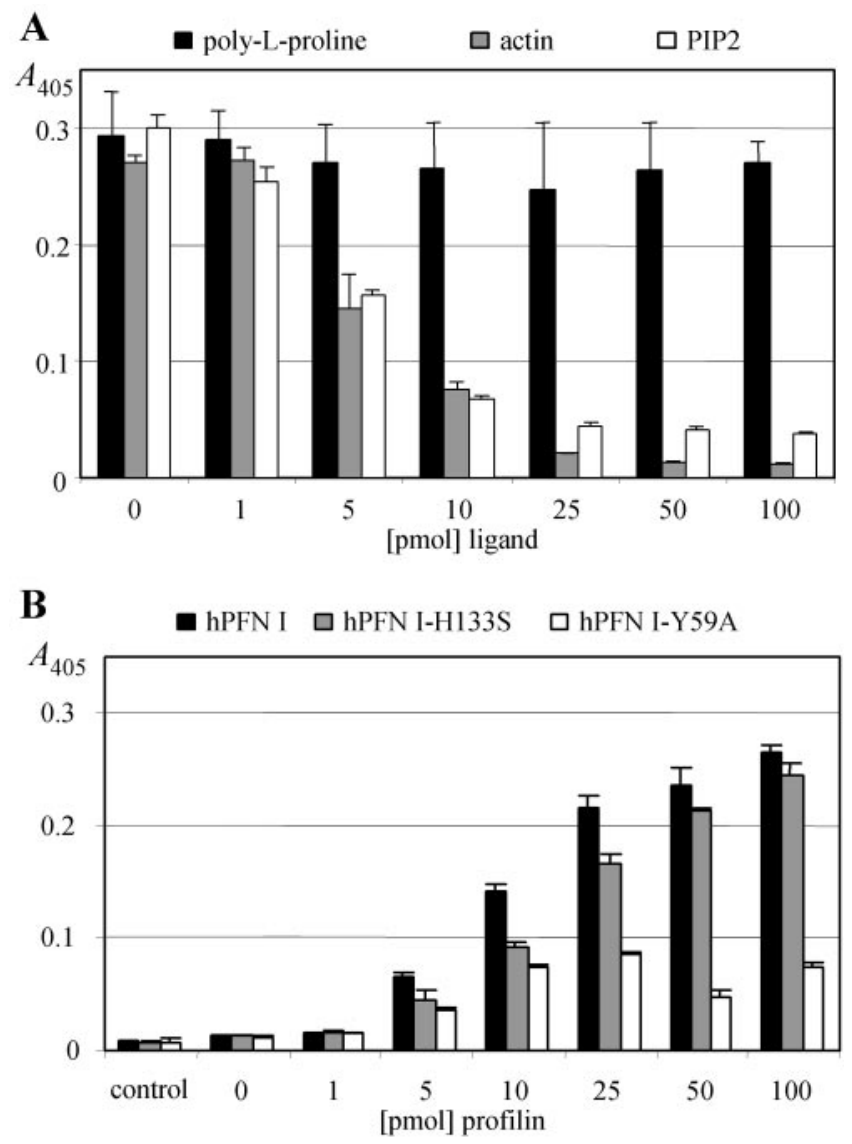

\section{C hPFN I- wt H133S Y59A

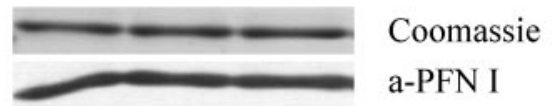

Figure 6. Gephyrin, $\mathrm{PIP}_{2}$, and $\mathrm{G}$-actin compete for the same binding site on profilins. $A$, ELISAs demonstrating the effects of poly-L-proline, actin, or $\mathrm{PIP}_{2}$ on the interaction of human profilin I with gephyrin. Constant amounts of profilin I ( $25 \mathrm{pmol})$, preincubated with increasing amounts of poly-L-proline, actin, or $\mathrm{PIP}_{2}$ (picomole ligands), were added to ELISA plates coated with 25 pmol of gephyrin. Binding of profilin was monitored with the monoclonal anti-profilin. Note that preincubation of profilin with poly-t-proline had no significant effect on binding to gephyrin, whereas actin and $\mathrm{PIP}_{2}$ led to a dose-dependent reduction of profilin binding. Because $\mathrm{PIP}_{2}$ was added in the form of micelles, the effects of actin and $\mathrm{PIP}_{2}$ on profilin binding to gephyrin cannot be compared in terms of affinities. Error bars indicate SD of three independent experiments. B, ELISAs showing binding of gephyrin to mutant profilins. Microwell-adsorbed recombinant gephyrin was incubated with increasing amounts of recombinant wild-type human profilin I, or profilin with a point mutation in the poly-proline binding site (hPFN I-H133S) or in the actin binding site ( $\mathrm{hPFN}$ I-Y59A), respectively. Binding was monitored with the monoclonal anti-profilin. Note that binding of hPFN I-H133S (gray bars) was decreased only slightly compared with hPFN I (black bars), whereas binding of hPFN I-Y59A (white bars) was significantly reduced. Control was $25 \mathrm{pmol}$ of microwell-adsorbed BSA incubated with $100 \mathrm{pmol}$ of the different profilins as indicated. $C$, Immunoblots of the profilin mutants and the same antibody used in $B$ to show that the immunoreactivity of all samples used is comparable.

polyproline remains essentially unaffected (Schlüter et al., 1998) (K. Schlüter and B. M. Jockusch, unpublished results). Conversely, replacement of a histidine residue within the human profilin I polyproline binding site (H133S) had been shown to reduce polyproline binding dramatically, whereas it only moderately affects actin-binding (Bjorkegren et al., 1993) (our unpublished results). As shown in Figure $6 B$, binding characteristics of wildtype human profilin I to microwell-adsorbed gephyrin approached saturation at a molar ratio of profilin to gephyrin of $4: 1$. 
The binding of the mutant hPFN I-H133S was reduced only slightly, whereas the mutation within the actin binding site, Y59A, led to a drastic decrease in the affinity for gephyrin. These results were not caused by differential recognition of the profilin variants by the monitoring antibody, as shown in immunoblots (Fig. 6C). Therefore, these findings confirm the data obtained from the competitive ELISA and support the notion that gephyrin interacts directly with the actin/ $\mathrm{PIP}_{2}$ binding site on profilin.

\section{Cytoplasmic gephyrin clusters are enriched in profilins, G-actin, and Mena/VASP}

The interaction of gephyrin with microfilament proteins was also analyzed by light microscopy in HeLa and PC12 cells transfected with green fluorescent protein (GFP)-gephyrin. The transfected cells displayed numerous gephyrin clusters (Fig. 7A-D), as described previously for HEK293 cells (Kirsch et al., 1995; Fuhrmann et al., 2002). As illustrated in Figure 7, $A$ and $A^{\prime \prime}$, doublefluorescence images revealed that Flag-profilin I was enriched in these structures. Identical results were obtained for endogenous profilin I and for transfected profilin IIa. Furthermore, in reciprocal experiments with BiPro-tagged gephyrin and GFP-profilin, we obtained the same results (data not shown). To rule out the possibility that these results were restricted to the two cell lines used, analogous experiments were performed with HEK293 cells and yielded identical results (data not shown).

A possible association of gephyrin clusters with additional microfilament proteins was also tested with transfected cells. Staining the GFP-gephyrin-transfected cells with TRITC-labeled phalloidin revealed prominent stress fibers, but no obvious association of actin filaments with gephyrin aggregates and no differences in stress fiber organization, as compared with nontransfected cells (data not shown); however, the clusters were found enriched in G-actin. Monomeric actin can be localized in cells after fixation with the cross-linker DSP, which preserves G-actin in its native conformation, and an antibody specifically recognizing this monomer configuration (Gonsior et al., 1999). Using this fixation and immunostaining protocol, we found that the gephyrin clusters are enriched in G-actin (Fig. $7 B, B^{\prime \prime}$ ).

Because we had shown that profilin, a component of the aggregates, cannot simultaneously bind to monomeric actin and gephyrin (Fig. 6), we probed the possibility of a direct interaction of gephyrin with G-actin. In gel filtration, ELISAs, and overlay experiments, we could not obtain convincing evidence for a gephyrin-G-actin interaction (data not shown) (Fig. $5 A^{\prime}$ ). Therefore, the enrichment of gephyrin clusters in G-actin suggested the participation of another component. The microfilament and focal adhesion protein VASP seemed a good candidate because it binds both to the polyproline-binding motif of profilins (Reinhard et al., 1995) and to G-actin (Walders-Harbeck et al., 2002) and nucleates actin filament assembly (Hüttelmaier et al., 1999). Indeed, immunofluorescence analysis of transfected HeLa cells with a VASP antibody revealed an enrichment of VASP in these clusters and a colocalization with GFP-gephyrin aggregates (Fig. $7 C, C^{\prime \prime}$ ). Furthermore, in $\mathrm{PC} 12$ cells, a paraneuronal cell line derived from phaeochromocytoma, the GFPgephyrin clusters were strongly enriched in the neuronal VASP homolog, Mena (Fig. $7 D, D^{\prime \prime}$ ).

\section{Gephyrin interacts directly with Mena/VASP}

A putative direct interaction of Mena/VASP and gephyrin was studied in ELISAs. Recombinant, microwell-adsorbed gephyrin was incubated with recombinant BiPro-tagged VASP, and binding was monitored with the BiPro antibody. As shown in Figure
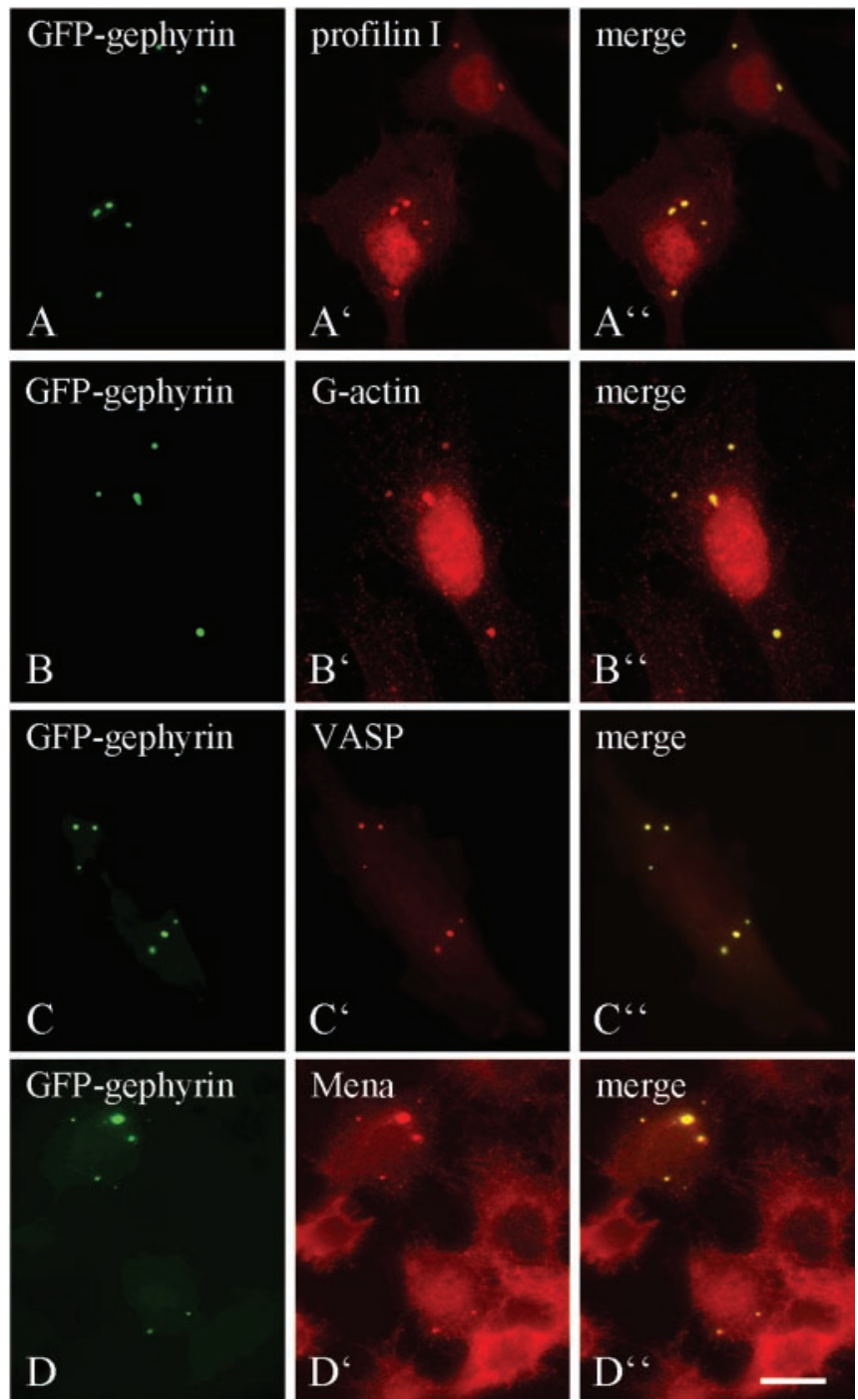

Figure 7. Profilin, $\mathrm{G}$-actin, and Mena/VASP are enriched in gephyrin clusters. HeLa ( $A-C$, $\left.A^{\prime \prime}-C^{\prime \prime}\right)$ and $P C 12\left(D, D^{\prime \prime}\right)$ cells were transiently transfected with pEGFP-gephyrin resulting in the formation of large cytoplasmic gephyrin clusters (shown by green autofluorescence in $A-D$ ). $A$, $A^{\prime \prime}$, Cells cotransfected with pFlag-mPFN I. Immunofluorescence with the Flag antibody shows profilin I $\left(A^{\prime}\right.$, red) in the GFP-gephyrin aggregates. $B, B^{\prime \prime}$, Immunolabeling with an antibody specific for monomeric actin ( $B^{\prime}$, red) demonstrates endogenous nuclear actin as described (cf. Gonsior et al., 1999) and cytoplasmic G-actin in small cytoplasmic particles or dots and G-actin enrichment in each of the gephyrin clusters. $C, C^{\prime \prime}$, Endogenous VASP was detected with a monoclonal antibody $\left(C^{\prime}\right.$, red) as an additional component of gephyrin clusters in HeLa cells. $D$, $D^{\prime \prime}$, Immunofluorescence of GFP-gephyrin-transfected PC12 cells showing that gephyrin clusters in these paraneuronal cells are decorated with an antibody against the neuronal VASP relative Mena $\left(D^{\prime}\right.$, red). Colocalization is demonstrated by superimposition of the corresponding images $\left(A^{\prime \prime}-D^{\prime \prime}\right)$. Scale bars, $10 \mu \mathrm{m}$.

$8 \mathrm{~A}$, binding occurred in a saturable manner, suggesting a $1: 1$ stoichiometry. We also probed for gephyrin-VASP interactions in a physiological environment. Immunoprecipitation studies with gephyrin-transfected HeLa cells showed that not only profilin (compare Fig. 5) but also HeLa cell VASP coprecipitated with Flag-tagged gephyrin or Flag-tagged E-domain (Fig. 8B). Furthermore, gephyrin and Mena could be coimmunoprecipitated from rat brain extracts. Figure $8 C$ shows that in immunoblots obtained with such precipitates, two of the three splice variants of Mena could be detected. One of these, the $140 \mathrm{kDa}$ large brain-specific isoform (Gertler et al., 1996) was preferentially precipitated. Analogous results were obtained in pull-down ex- 


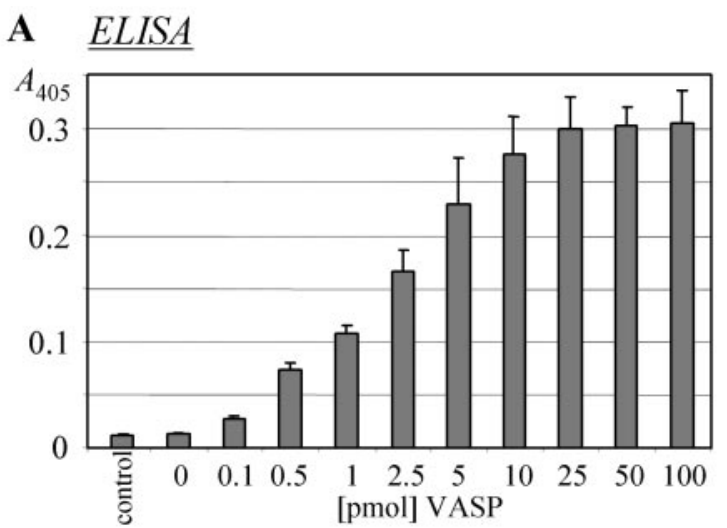

B Co-IP from HeLa-cells:

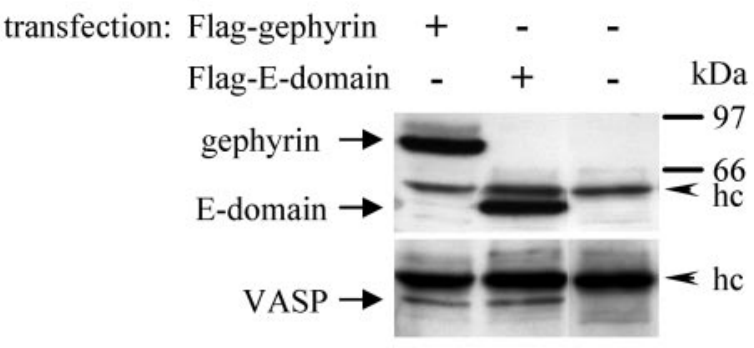

C Co-IP from rat brain:

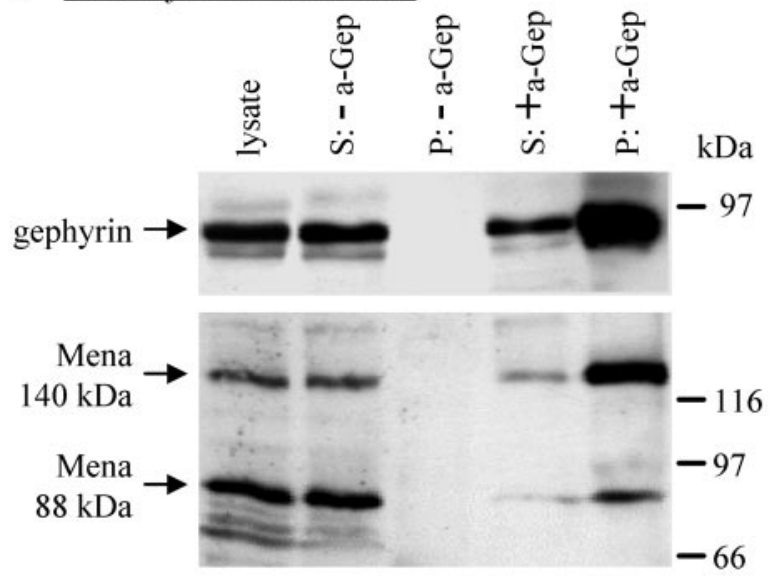

Figure 8. Gephyrin and Mena/VASP interact in vitro and form complexes in cells. A, ELISA to show direct interaction between gephyrin and VASP. Microwells were coated with $25 \mathrm{pmol}$ of recombinant gephyrin and incubated with increasing amounts of recombinant BiPro-VASP as indicated. The BiProantibody was used to monitor binding. Note that binding of VASP to gephyrin reaches saturation level at a 1:1 molar ratio. Control was $25 \mathrm{pmol}$ of BSA, microwell adsorbed, instead of gephyrin. $B$, Coimmunoprecipitation of VASP with gephyrin or the isolated E-domain. Immunoprecipitates obtained from HeLa cells transiently expressing Flag-tagged gephyrin or the Flag-tagged E-domain were obtained with the Flag antibody and subjected to SDS-PAGE and immunoblotting. Antibodies against Flag and VASP, respectively, revealed the transfected gephyrin or the E-domain and VASP in the precipitate, whereas VASP was not sedimented from nontransfected cells. Note that the E-domain of gephyrin is sufficient for binding to VASP. hc, Heavy chain of the precipitating lgG. C, Coimmunoprecipitation of Mena and gephyrin from rat brain extracts, performed with ( + a-Gep) or without $(-a-$ Gep) polyclonal anti-gephyrin. The immunoblots obtained with Mena antibodies from anti-gephyrininduced precipitates reveal that two of the three Mena splice variants ( 88 and $140 \mathrm{kDa}$ ) are detectable in the sediment. Note that the large $140 \mathrm{kDa}$ brain-specific isoform is preferentially enriched in the complex.

periments from rat spinal cord lysates (data not shown). Together with the data presented in Figure 3, these results imply that gephyrin, profilin, and Mena/VASP can be present in the same complexes.
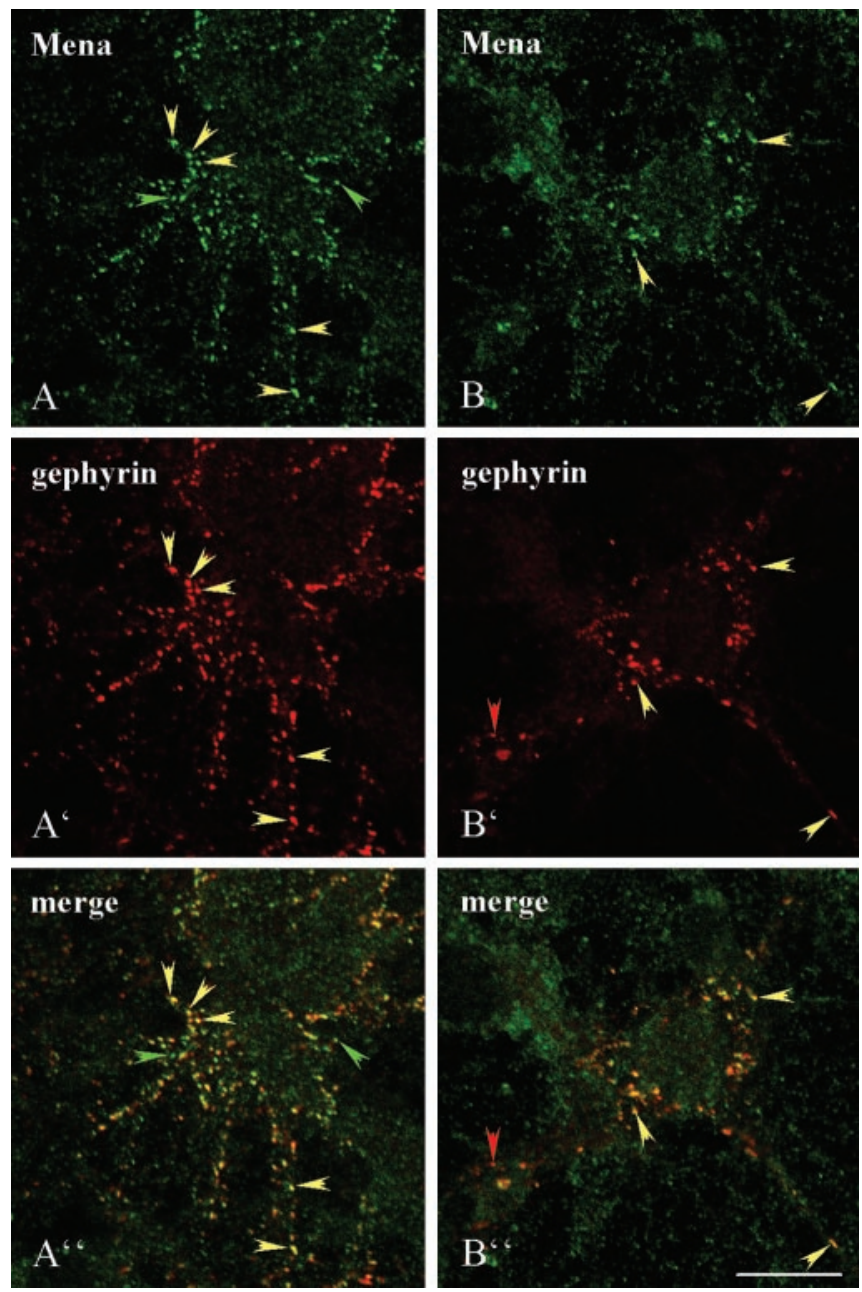

Figure 9. Colocalization of Mena with synaptic structures in cultured spinal cord neurons. Double immunofluorescence for Mena $\left(A, B\right.$, green) and gephyrin $\left(A^{\prime}, B^{\prime}\right.$, red). The merged images $\left(A^{\prime \prime}, B^{\prime \prime}\right)$ show that most, but not all, synapses contain both proteins. Yellow arrowheads indicate examples of colocalization; green and red arrowheads indicate spots only positive for Mena and gephyrin, respectively. Scale bars, $10 \mu \mathrm{m}$.

The association of gephyrin with Mena was studied further in cultured rat spinal cord neurons (Fig. 9). Immunofluorescence showed Mena concentrated in speckles distributed at the periphery of the cell body as well as in dendrites (Fig. 9A,B). Counterstaining for gephyrin identified these immunoreactive spots as inhibitory synapses (Fig. $\left.9 A^{\prime}, B^{\prime}\right)$. Merged images revealed a high degree of colocalization of Mena and gephyrin (Fig. $9 A^{\prime \prime}, B^{\prime \prime}$ ).

\section{Discussion}

In this report, we present cell biological, biochemical, and immunohistochemical evidence for the postulated molecular interaction between gephyrin and the actin-based cytoskeleton. Such links have been suggested previously from experiments demonstrating that interference with submembranous microfilament integrity has severe consequences on receptor clustering that in turn depends on gephyrin scaffolds (Kirsch and Betz, 1995). We identified the microfilament-associated proteins profilin, Mena/ VASP, and G-actin as partners for gephyrin in tissue, cells, and in vitro. Complex formation between gephyrin and profilin I had been reported before and was thought to be mediated by the proline-rich central region on gephyrin and the polyprolinebinding motif on profilin (Mammoto et al., 1998). In this case 
one would have expected profilin IIa, the neuronal isoform, to bind with higher affinity to gephyrin than profilin I (cf. Lambrechts et al., 1997). Because we did not detect such a difference, this mode of interaction seemed unlikely. Instead, we identified gephyrin as a ligand for the actin/ $\mathrm{PIP}_{2}$ binding site on profilin and both profilin isoforms as binding partners for the E-domain of gephyrin. This large gephyrin module also accommodates a binding motif for the microfilament adaptor proteins of the Mena/ VASP family. Complexes containing gephyrin, profilin, and Mena/VASP may thus exist in neuronal tissue and cells. They may form by various interactions, either by profilin binding gephyrin and Mena/VASP or by Mena/VASP bridging gephyrin to profilin. Furthermore, a direct interaction of profilin and Mena/VASP with discrete binding sites on the E- domain of gephyrin is also possible. G-actin may join these complexes by binding to the corresponding motif on Mena/VASP (Walders-Harbeck et al., 2002) but may also be bound to profilin. In the latter case, a competition between G-actin and gephyrin for the same binding site on profilin, as evident from our in vitro data, may add another variant to the multiple modes of molecular communication and complex formation. Such variability would be important for the fine regulation of the amount of polymerization-competent actin available for de novo generation of actin filaments in a submembranous microfilament web. A simultaneous anchoring of gephyrin clusters to microfilaments and microtubules, as postulated previously (Kirsch and Betz, 1995), is also conceivable, because the tubulin-binding motif and the binding site for the light chains of the microtubule motor protein dynein are both located in the central domain of gephyrin (Kirsch et al., 1991; Fuhrmann et al., 2002), whereas the data presented here define the E-domain as a molecular link to the microfilament system.

Linkage of gephyrin to a cortical microfilament web resulting in a high and precise packing density of gephyrin scaffolds might be of functional significance for both neuronal and non-neuronal cells. In addition to restricting lateral mobility of the neuronal receptors as outlined above, an analogous submembranous concentration of gephyrin by microfilaments should also be advantageous for molybdenum cofactor synthesis and substrate channeling in all cell types (Schwarz et al., 2001). Furthermore, profilin and Mena/VASP, the gephyrin ligands identified here, are considered essential and ubiquitous components of cortical microfilament webs that in general govern the mobility of many membrane-associated or transmembrane proteins. Within this submembranous compartment, profilins are thought to regulate actin polymerization in a $\mathrm{PIP}_{2}$-dependent manner (cf. Schlüter et al., 1997), whereas Mena/VASP proteins may increase the efficiency of actin polymerization by recruiting profilin and G-actin and contribute to the arrangement of cortical microfilaments by nucleating actin filament formation and bundling (Hüttelmaier et al., 1999; Lambrechts et al., 2000; Walders-Harbeck et al., 2002). A role for Mena/VASP in transversal plasma membrane motility, in particular during the actin-driven protrusion of lamellipodia, is well established (Cramer, 2002), but an additional function in regulating lateral submembranous protein mobility is also quite likely. Whatever the precise mechanism, there is no doubt that Mena/VASP proteins are important for neuronal positioning during embryogenesis (Goh et al., 2002). Menadeficient mice that are heterozygous for a profilin I deletion die before birth, showing defects of neurulation (Lanier et al., 1999). This demonstrates that both profilin I and Mena play an important role in neuronal cells. On the basis of findings reported here, both proteins, in conjunction with gephyrin, may specifically reg- ulate the stabilization and activity-dependent adaptation of inhibitory synapses.

\section{References}

Bear JE, Svitkina TM, Krause M, Schafer DA, Loureiro JJ, Strasser GA, Maly IV, Chaga OY, Cooper JA, Borisy GG, Gertler FB (2002) Antagonism between Ena/VASP proteins and actin filament capping regulates fibroblast motility. Cell 109:509-521.

Bjorkegren C, Rozycki M, Schutt CE, Lindberg U, Karlsson R (1993) Mutagenesis of human profilin locates its poly(L-proline)-binding site to a hydrophobic patch of aromatic amino acids. FEBS Lett 333:123-126.

Carlsson L, Nystrom L, Sundkvist I, Markey F, Lindberg U (1976) Profilin, a low-molecular weight protein controlling actin polymerisability. In: Contractile systems in non-muscle tissues (Perry SV, Margreth A, Adelstein RS, eds), pp 39-49. Amsterdam: North Holland.

Cramer LP (2002) Ena/Vasp: solving a cell motility paradox. Curr Biol 12:R417-R419.

Di Nardo A, Gareus R, Kwiatkowski D, Witke W (2000) Alternative splicing of the mouse profilin II gene generates functionally different profilin isoforms. J Cell Sci 113:3795-3803.

Feng G, Tintrup H, Kirsch J, Nichol MC, Kuhse J, Betz H, Sanes JR (1998) Dual requirement for gephyrin in glycine receptor clustering and molybdoenzyme activity. Science 282:1321-1324.

Fuhrmann JC, Kins S, Rostaing P, El Far O, Kirsch J, Sheng M, Triller A, Betz H, Kneussel M (2002) Gephyrin interacts with dynein light chains 1 and 2, components of motor protein complexes. J Neurosci 22:5393-5402.

Gertler FB, Niebuhr K, Reinhard M, Wehland J, Soriano P (1996) Mena, a relative of VASP and Drosophila enabled, is implicated in the control of microfilament dynamics. Cell 87:227-239.

Giehl K, Valenta R, Rothkegel M, Ronsiek M, Mannherz HG, Jockusch BM (1994) Interaction of plant profilin with mammalian actin. Eur J Biochem 226:681-689.

Giesemann T, Rathke-Hartlieb S, Rothkegel M, Bartsch JW, Buchmeier S, Jockusch BM, Jockusch H (1999) A role for polyproline motifs in the spinal muscular atrophy protein SMN: profilins bind to and colocalize with SMN in nuclear gems. J Biol Chem 274:37908-37914.

Goh KL, Cai L, Cepko CL, Gertler FB (2002) Ena/VASP proteins regulate cortical neuronal positioning. Curr Biol 12:565-569.

Gonsior SM, Platz S, Buchmeier S, Scheer U, Jockusch BM, Hinssen H (1999) Conformational difference between nuclear and cytoplasmic actin as detected by a monoclonal antibody. J Cell Sci 112:797-809.

Harbeck B, Hüttelmaier S, Schlüter K, Jockusch BM, Illenberger S (2000) Phosphorylation of the vasodilator-stimulated phosphoprotein regulates its interaction with actin. J Biol Chem 275:30817-30825.

Honoré B, Madsen P, Andersen AH, Leffers H (1993) Cloning and expression of a novel human profilin variant, profilin II. FEBS Lett 330:151-155.

Hüttelmaier S, Mayboroda O, Harbeck B, Jarchau T, Jockusch BM, Rüdiger M (1998) The interaction of the cell-contact proteins VASP and vinculin is regulated by phosphatidylinositol-4,5-bisphosphate. Curr Biol 8:479-488.

Hüttelmaier S, Harbeck B, Steffens O, Messerschmidt T, Illenberger S, Jockusch BM (1999) Characterization of the actin binding properties of the vasodilator-stimulated phosphoprotein VASP. FEBS Lett 451:68-74.

Kirsch J, Betz H (1993) Widespread expression of gephyrin, a putative glycine receptor-tubulin linker protein, in rat brain. Brain Res 621:301-310.

Kirsch J, Betz H (1995) The postsynaptic localization of the glycine receptor-associated protein gephyrin is regulated by the cytoskeleton. J Neurosci 15:4148-4156.

Kirsch J, Langosch D, Prior P, Littauer UZ, Schmitt B, Betz H (1991) The 93-kDa glycine receptor-associated protein binds to tubulin. J Biol Chem 266:22242-22245.

Kirsch J, Kuhse J, Betz H (1995) Targeting of glycine receptor subunits to gephyrin-rich domains in transfected human embryonic kidney cells. Mol Cell Neurosci 6:450-461.

Kneussel M, Betz H (2000) Clustering of inhibitory neurotransmitter receptors at developing postsynaptic sites: the membrane activation model. Trends Neurosci 23:429-435.

Kneussel M, Brandstatter JH, Laube B, Stahl S, Muller U, Betz H (1999) Loss of postsynaptic GABA(A) receptor clustering in gephyrin-deficient mice. J Neurosci 19:9289-9297.

Lambrechts A, Verschelde JL, Jonckheere V, Goethals M, Vandekerckhove J, 
Ampe C (1997) The mammalian profilin isoforms display complementary affinities for PIP2 and proline-rich sequences. EMBO J 16:484-494.

Lambrechts A, Kwiatkowski AV, Lanier LM, Bear JE, Vandekerckhove J, Ampe C, Gertler FB (2000) cAMP-dependent protein kinase phosphorylation of EVL, a Mena/VASP relative, regulates its interaction with actin and SH3 domains. J Biol Chem 275:36143-36151.

Lanier LM, Gates MA, Witke W, Menzies AS, Wehman AM, Macklis JD, Kwiatkowski D, Soriano P, Gertler FB (1999) Mena is required for neurulation and commissure formation. Neuron 22:313-325.

Mammoto A, Sasaki T, Asakura T, Hotta I, Imamura H, Takahashi K, Matsuura Y, Shirao T, Takai Y (1998) Interactions of drebrin and gephyrin with profilin. Biochem Biophys Res Commun 243:86-89.

Mayboroda O, Schlüter K, Jockusch BM (1997) Differential colocalization of profilin with microfilaments in PtK2 cells. Cell Motil Cytoskeleton 37:166-177.

Meier J, Vannier C, Serge A, Triller A, Choquet D (2001) Fast and reversible trapping of surface glycine receptors by gephyrin. Nat Neurosci 4:253-260.

Meyer G, Kirsch J, Betz H, Langosch D (1995) Identification of a gephyrin binding motif on the glycine receptor beta subunit. Neuron 15:563-572.

Pfeiffer F, Graham D, Betz H (1982) Purification by affinity chromatography of the glycine receptor of rat spinal cord. J Biol Chem 257:9389-9393.

Prior P, Schmitt B, Grenningloh G, Pribilla I, Multhaup G, Beyreuther K, Maulet Y, Werner P, Langosch D, Kirsch J, et al (1992) Primary structure and alternative splice variants of gephyrin, a putative glycine receptor-tubulin linker protein. Neuron 8:1161-1170.

Ramming M, Kins S, Werner N, Hermann A, Betz H, Kirsch J (2000) Diversity and phylogeny of gephyrin: tissue-specific splice variants, gene structure, and sequence similarities to molybdenum cofactor-synthesizing and cytoskeleton-associated proteins. Proc Natl Acad Sci USA 97:10266-10271.

Reinhard M, Halbrugge M, Scheer U, Wiegand C, Jockusch BM, Walter U (1992) The $46 / 50 \mathrm{kDa}$ phosphoprotein VASP purified from human platelets is a novel protein associated with actin filaments and focal contacts. EMBO J 11:2063-2070.

Reinhard M, Giehl K, Abel K, Haffner C, Jarchau T, Hoppe V, Jockusch BM, Walter U (1995) The proline-rich focal adhesion and microfilament protein VASP is a ligand for profilins. EMBO J 14:1583-1589.

Rüdiger M, Jockusch BM, Rothkegel M (1997) An epitope tag-antibody combination useful for the detection of protein expression in procaryotic and eukaryotic cells. BioTechniques 23:96-97.

Schlüter K, Jockusch BM, Rothkegel M (1997) Profilins as regulators of actin dynamics. Biochim Biophys Acta 27:97-109.

Schlüter K, Schleicher M, Jockusch BM (1998) Effects of single amino acid substitutions in the actin-binding site on the biological activity of bovine profilin I. J Cell Sci 111:3261-3273.

Schmitt B, Knaus P, Becker CM, Betz H (1987) The $M_{\mathrm{r}} 93,000$ polypeptide of the postsynaptic glycine receptor complex is a peripheral membrane protein. Biochemistry 26:805-811.

Schwarz G, Schrader N, Mendel RR, Hecht HJ, Schindelin H (2001) Crystal structures of human gephyrin and plant Cnx1 G domains: comparative analysis and functional implications. J Mol Biol 312:405-418.

Stallmeyer B, Schwarz G, Schulze J, Nerlich A, Reiss J, Kirsch J, Mendel RR (1999) The neurotransmitter receptor-anchoring protein gephyrin reconstitutes molybdenum cofactor biosynthesis in bacteria, plants, and mammalian cells. Proc Natl Acad Sci USA 96:1333-1338.

Walders-Harbeck B, Khaitlina SY, Hinssen H, Jockusch BM, Illenberger S (2002) The vasodilator-stimulated phosphoprotein promotes actin polymerisation through direct binding to monomeric actin. FEBS Lett 26564:1-6.

Wittenmayer N, Rothkegel M, Jockusch BM, Schlüter K (2000) Functional characterization of green fluorescent protein-profilin fusion proteins. Eur J Biochem 267:5247-5256. 